\title{
Structure of multi-component/multi-Yukawa mixtures
}

\section{Blum $\uparrow \S$ and M. Arias $\dagger$}

$\dagger$ Department of Physics, P.O. Box 23343, University of Puerto Rico, Rio Piedras, PR 00931-3343 and Department of Mathematics, Hill Center Rutgers University, Piscataway N.J. 08854

\begin{abstract}
Recent small angle scattering experiments reveal new peaks in the structure function $S(k)$ of colloidal systems ( S.H. Chen et al [1]), in a region that was inaccessible with older instruments. It has been increasingly evident that a single (or double) Yukawa MSA-closures cannot account for these observations, and three or more terms are needed. On the other hand the MSA is not sufficiently accurate 2] more accurate theories such as the HNC have been tried. But while the MSA is asymptotically exact at high densities $[3]$ it does not satisfy the low density asymptotics. This has been corrected in the soft-MSA [4, 5] by adding exponential type terms. The results compared to experiment and simulation for liquid sodium by Rahman and Paskin ( as shown in 4) are remarkably good. We use here a general closure of the Ornstein Zernike equation which is not necessarily the MSA closure [6].

$$
c_{i j}(r)=\sum_{n=1}^{M} \mathcal{K}_{i j}^{(n)} e^{-z_{n} r} / r \quad \mathcal{K}_{i j}^{(n)}=K^{(n)} \delta_{i}^{(n)} \delta_{j}^{(n)} ; \quad r \geq \sigma_{i j}
$$
\end{abstract}

with the boundary condition for $g_{i j}(r)=0$ for $r \leq \sigma_{i j}$. This general closure of the Ornstein Zernike equation will go well beyond the MSA since it has been tested by Monte Carlo simulation for tetrahedral water [7, toroidal ion channels [8] and polyelectrolytes $[9$. For this closure we get for the Laplace transform of the pair correlation function an explicitly symmetric result

$$
2 \pi \tilde{g}_{i j}(s)=-\frac{e^{-s \sigma_{i j}}}{D_{\tau}(s)}\left\{\frac{1}{s^{2}}+\frac{1}{s} Q_{i j}^{\prime}\left(\sigma_{i j}\right)+\sum_{m=1}^{M} \frac{z_{m} \tilde{\mathcal{X}}_{i}^{(m)} f_{j}^{(m)}}{s+z_{m}}\right\}
$$

This function is also easily transformed into $S(k)$ by replacing $s \Rightarrow i k$. For low density situations (dilute colloids) $D_{\tau}(s) \sim 1+\mathcal{O}(\rho)$ and the $S(k)$ is a sum of $M$ Lorentzians. For hard sphere PY mixtures we get the simple (compare [10, 11])

$$
2 \pi \tilde{g}_{i j}(s)=-\frac{e^{-s \sigma_{i j}}}{s^{2} D_{\tau}(s)}\left\{1+s\left[\left(Q^{H S}\right)_{i j}^{\prime}\left(\sigma_{i j}\right)\right]\right\}
$$

where $D_{\tau}(s)$ is a scalar function. For polydisperse electrolytes in the MSA a simpler expression is also obtained (compare [12]) . An explicit continued fraction solution of the 1 component multiyukawa case is also given.

PACS numbers: 61.20.Gy, 82.70.Dd, 61.12.Ex, 61.20.Gy, 61.20.Ja

$\S$ To whom correspondence should be addressed (lesblum@yahoo.com) 
16 August 2018, yuksowhsinv3a, ArXiv cond-mat/0602477 IOP : CM/219099/SPE/8462 Submitted to: J. Phys.: Condens. Matter 


\section{Introduction}

There are many problems of practical and academic interest that can be formulated as closures of some kind of either scalar or matrix Ornstein-Zernike (OZ) equation. These closures can always be expressed by a sum of exponentials, which do form a complete basis set if we allow for complex numbers [13, 14. It is of practical interest to be able to relate small angle scattering experiments as directly as posible to theoretical (molecular) parameters. For a number of systems the MSA ([13, 15, 16]) has been generally adequate (11, 17]). The GOCM which is a the single peak-single Yukawa closure-MSA description is adequate in the simple cases. However, recent high resolution experiments have shown that to account for the additional peaks seen in the SANS experiments more yukawas are required [2, 18, 19]. The MSA and HNC closures of the OZ equation slightly underestimate the height of the interaction peak in the structure factor, whereas a close comparison of the cluster peak position is somewhat hindered by the limited resolution in $\mathrm{Q}$ of the simulation data, because the largest simulation box is never big enough. In most part of the practical range of potential parameters, the theoretical predictions coming from the two closures have comparable accuracy. Therefore, for fitting the neutron scattering intensity distributions, analytical structure factors are preferable since the resolution of the equations is more stable and fast.

Our present solution makes the direct comparison between theory and experiment feasible since a simple expression for the Fourier transform $S(Q)$ is proposed which is directly related to the closure of the OZ equation. In fact with the very mild assumption made is that the direct correlation function can be expanded as

$$
c_{i j}(r)=\sum_{n=1}^{M} K_{i j}^{(n)} e^{-z_{n}\left(r-\sigma_{i j}\right)} / r=\sum_{n=1}^{M} \mathcal{K}_{i j}^{(n)} e^{-z_{n} r} / r ; \quad r>\sigma_{i j}
$$

In the factorizable case (which is also the electrostatic charge case), we have

$$
K_{i j}^{(n)}=K^{(n)} \delta_{i}^{(n)} \delta_{j}^{(n)} ; \quad \mathcal{K}_{i j}^{(n)}=K^{(n)} d_{i}^{(n)} d_{j}^{(n)} ; \quad \delta_{i}^{(n)}=d_{i}^{(n)} e^{-z_{n} \sigma_{i} / 2}
$$

with

$$
g_{i j}(r)=0 \quad \text { for } \quad r \leq \sigma_{i j}
$$

Here $z_{n}$ can be a complex number, and then eq.(3) is a complete function basis set. This closure works very well with a number of potentials, that include Lennard-Jones, Buckingham, and liquid metals [4, 20]. The effective interaction among charged colloids and globular proteins in solution is also well described by this potential which is the sum of a short range part attraction, usually accounting for van der Waals or entropic forces, plus a long range repulsion (of up to a few particle diameters), arising from screened electrostatic potential[21]. The two-Yukawa (2Y) fluid, consisting of particles interacting with this potential can be usefully applied to a wide variety of systems such as C60 fullerenes at high temperature 22, globular proteins [23, 24], and the DerjaguinLandau- Verwey-Overbeek (DLVO) 25] potential for liophobic colloids. 
While the initial motivation was to study simple approximations like the Mean Spherical (MSA) or Generalized Mean Spherical Approximation (GMSA), the availability of closed form solutions for the general closure of the hard core OZ equation makes it possible to write down analytical solutions for any given approximation that can be formulated by writing the direct correlation function $c(r)$ outside the hard core as

$$
c(r)=\sum_{n=1}^{M} K^{(n)} e^{-z_{n}(r-\sigma)} / r=\sum_{n=1}^{M} \mathcal{K}^{(n)} e^{-z_{n} r} / r
$$

In this equation $K^{(n)}$ is the interaction/closure constant used in the general solution first found by Blum and Hoye (which we will call BH78) [20, while $\mathcal{K}^{(n)}$ is the definition used in the later general solution by Blum, Vericat and Herrera (BVH92 in what follows) [26]. In this work we will use the more common notation of BVH92. The case of factored interactions was discussed by Blum, [27, 28, and by Ginoza [29, 30, 31, 32. The general solution of this problem [26] is given in terms of the scaling matrix $\boldsymbol{\Gamma}$ which will comply with the physical constraints of the systems (Blum et al. 14, 33, 34). The solution of the resulting algebraic equations has a number of branches (Pastore [35]). The rigorous analysis of this question is very complex. We have used the following working hypothesis:

(i) The singularities of the equations are poles, branch cuts and essential (exponential) sigularities.

(ii) The physical branch is the one for which the correct zero density result is obtained. This is equivalent to what is done in the rigorous treatment van der Waals theory, and includes metastable regions and spinodals.

(iii) The analysis of the repulsive part of the potential is more delicate since it is clearly related to the exponential singularities, and the convexity of the limiting high density configurations [36].

For only one component the matrix $\boldsymbol{\Gamma}$ can be assumed to be diagonal without loss of generality, and explicit expressions for the closure relations for any arbitrary number of Yukawa exponents $M$ were obtained. The solution is then remarkably simple in the MSA since then explicit formulas for the thermodynamic properties are obtained. However there are undetermined integration constants in the entropy which have to be adjusted to get the correct limiting behavior [17].

\section{Summary of Previous Work}

We study the Ornstein-Zernike (OZ) equation

$$
h_{i j}(12)=c_{i j}(12)+\sum_{k} \int d 3 h_{i k}(13) \rho_{k} c_{k j}(32)
$$

where $h_{i j}(12)$ is the molecular total correlation function and $c_{i j}(12)$ is the molecular direct correlation function, $\rho_{i}$ is the number density of the molecules $\mathrm{i}$, and $i=1,2$ is 
Structure of multi-component/multi-Yukawa mixtures

the position $\vec{r}_{i}, r_{12}=\left|\vec{r}_{1}-\vec{r}_{2}\right|$ and $\sigma_{i j}$ is the distance of closest approach of two particles $i, j$. The direct correlation function is

$$
c_{i j}(r)=\sum_{n=1}^{M} K_{i j}^{(n)} e^{-z_{n}\left(r-\sigma_{i j}\right)} / r, \quad r>\sigma_{i j}
$$

and the pair correlation function is

$$
h_{i j}(r)=g_{i j}(r)-1=-1, \quad r \leq \sigma_{i j}
$$

We use the Baxter-Wertheim (BW) factorization of the OZ equation

$$
[\mathbf{I}+\rho \mathbf{H}(\mathbf{k})][\mathbf{I}-\rho \mathbf{C}(k)]=\mathbf{I}
$$

where $I$ is the identity matrix, and we have used the notation

$$
\begin{aligned}
& \mathbf{H}(k)=2 \int_{0}^{\infty} d r \cos (k r) \mathbf{J}(r) \\
& \mathbf{C}(k)=2 \int_{0}^{\infty} d r \cos (k r) \mathbf{S}(r)
\end{aligned}
$$

The matrices $J$ and $S$ have matrix elements

$$
\begin{aligned}
& J_{i j}(r)=2 \pi \int_{r}^{\infty} d \operatorname{ssh}_{i j}(s) \\
& S_{i j}(r)=2 \pi \int_{r}^{\infty} \operatorname{dssc}_{i j}(s) \\
& {[\mathbf{I}-\rho \mathbf{C}(k)]=[\mathbf{I}-\rho \mathbf{Q}(k)]\left[\mathbf{I}-\rho \mathbf{Q}^{T}(-k)\right]}
\end{aligned}
$$

where $\mathbf{Q}^{T}(-k)$ is the complex conjugate and transpose of $\mathbf{Q}(k)$. The first matrix is non-singular in the upper half complex $k$-plane, while the second is non-singular in the lower half complex $k$-plane.

It can be shown that the factored correlation functions must be of the form

$$
\mathbf{Q}(k)=\mathbf{I}-\rho \int_{\lambda_{j i}}^{\infty} d r e^{i k r} \mathbf{Q}(r)
$$

where we used the following definition

$$
\begin{aligned}
& \lambda_{j i}=\frac{1}{2}\left(\sigma_{j}-\sigma_{i}\right) \\
& \mathbf{S}(r)=\mathbf{Q}(r)-\int d r_{1} \mathbf{Q}\left(r_{1}\right) \rho \mathbf{Q}^{\mathbf{T}}\left(r_{1}-r\right)
\end{aligned}
$$

Similarly, from Eq. (14) and Eq. (9) we get, using the analytical properties of $Q$ and Cauchy's theorem

$$
\mathbf{J}(r)=\mathbf{Q}(r)+\int d r_{1} \mathbf{J}\left(r-r_{1}\right) \rho \mathbf{Q}\left(r_{1}\right)
$$

The general solution is discussed in [27, 29, 34], and yields

$$
\begin{aligned}
& q_{i j}(r)=q_{i j}^{0}(r)+\sum_{n=1}^{M} D_{i j}^{(n)} e^{-z_{n} r} \quad \lambda_{j i}<r \\
& q_{i j}^{0}(r)=(1 / 2) A_{j}\left[\left(r-\sigma_{j} / 2\right)^{2}-\left(\sigma_{i} / 2\right)^{2}\right]+\beta_{j}\left[\left(r-\sigma_{j} / 2\right)-\left(\sigma_{i} / 2\right)\right] \\
& +\sum_{n=1}^{M} C_{i j}^{(n)} e^{-z_{n} \sigma_{j} / 2}\left[e^{-z_{n}\left(r-\sigma_{j} / 2\right)}-e^{-z_{n} \sigma_{i} / 2}\right] \quad \lambda_{j i}<r<\sigma_{i j}
\end{aligned}
$$

The parameters $A_{j}, \beta_{j}, C_{i j}, D_{i j}$ are defined below and in Appendix 1 . 
Structure of multi-component/multi-Yukawa mixtures

\section{The Laplace Transforms and the structure functions}

From Eq. (18) we obtain the Laplace transform of the pair correlation function

$$
2 \pi \sum_{\ell} \tilde{g}_{i \ell}(s)\left[\delta_{\ell j}-\rho_{\ell} \tilde{q}_{\ell j}(i s)\right]=\tilde{q}_{i j}^{0^{\prime}}(i s)
$$

where

$$
\begin{aligned}
\tilde{q}_{i j}^{0^{\prime}}(i s) & =\int_{\sigma_{i j}}^{\infty} d r e^{-s r}\left[q_{i j}^{0}(r)\right]^{\prime} \\
= & {\left[\left(1+s \sigma_{i} / 2\right) A_{j}+s \beta_{j}\right] e^{-s \sigma_{i j}} / s^{2}-\sum_{m} \frac{z_{m}}{s+z_{m}} e^{-\left(s+z_{m}\right) \sigma_{i j}} C_{i j}^{(m)} }
\end{aligned}
$$

and

$$
\begin{aligned}
& e^{s \lambda_{j i}} \tilde{q}_{i j}(i s)=\sigma_{i}^{3} \psi_{1}\left(s \sigma_{i}\right) A_{j}+\sigma_{i}^{2} \phi_{1}\left(s \sigma_{i}\right) \beta_{j}+ \\
& \sum_{m} \frac{1}{s+z_{m}}\left[\left(C_{i j}^{(m)}+D_{i j}^{(m)}\right) e^{-z_{m} \lambda_{j i}}-C_{i j}^{(m)} e^{-z_{m} \sigma_{j i}}-z_{m} \sigma_{i} \phi_{0}\left(s \sigma_{i}\right) C_{i j}^{(m)} e^{-z_{m} \sigma_{j i}}\right]
\end{aligned}
$$

where

$$
\begin{aligned}
& \psi_{1}(x)=\left[1-x / 2-(1+x / 2) e^{-x}\right] /\left(x^{3}\right)=\frac{\sigma^{2}}{2 z} e^{-z \sigma / 2} i_{1}(z \sigma / 2) \\
& i_{1}(x)=\frac{1}{x^{2}}(\sinh x-x \cosh x) \\
& \phi_{1}(x)=\left[1-x-e^{-x}\right] /\left(x^{2}\right)=x \psi_{1}(x)-\phi_{0}(x) / 2 ; \quad \phi_{0}(x)=\frac{\left[1-e^{-x}\right]}{x}
\end{aligned}
$$

In the factored case (see eq(4I))

$$
D_{i j}^{(m)}=-\delta_{i}^{(m)} a_{j}^{(m)} ; \quad C_{i j}^{(m)}=\left(\delta_{i}^{(m)}-\frac{\mathcal{B}_{i}^{(m)}}{z_{m}}\right) a_{j}^{(m)} ; \quad \mathcal{B}_{i}^{(m)}=2 \pi \sum_{k} \rho_{k} g_{i k}^{(m)} \delta_{k}^{(m)}
$$

We remember that 26 ]

$$
\begin{aligned}
2 \pi \sigma_{i j} g_{i j}\left(\sigma_{i j}\right) & =q_{i j}^{\prime}\left(\sigma_{j i}^{-}\right)-q_{i j}^{\prime}\left(\sigma_{j i}^{+}\right) \\
& =A_{j}\left(\sigma_{i} / 2\right)+\beta_{j}-\sum_{m=1}^{M}\left(z_{m} \delta_{i}^{(m)}-\mathcal{B}_{i}^{(m)}\right) a_{j}^{(m)} e^{-z_{m} \sigma_{i j}}
\end{aligned}
$$

and

$$
\begin{aligned}
& \tilde{q}_{i j}(i s)=e^{-s \lambda_{j i}} \sigma_{i}^{3} \psi_{1}\left(s \sigma_{i}\right) A_{j}+e^{-s \lambda_{j i}} \sigma_{i}^{2} \phi_{1}\left(s \sigma_{i}\right) \beta_{j}- \\
& \sum_{m} \frac{e^{-z_{m} \lambda_{j i}}}{s+z_{m}} \quad\left(\delta_{i}^{(m)}+\left(\hat{\mathcal{B}}_{i}^{(m)}-\delta_{i}^{(m)}\right)\left\{1-e^{-\left(z_{m}+s\right) \sigma_{i}}\left[1+z_{m} \sigma_{i} \phi_{0}\left(\sigma_{i} s\right)\right]\right\}\right) a_{j}^{(m)}
\end{aligned}
$$


which can be written in the form

$$
\begin{aligned}
\mathbf{M}_{i j}(s)=\delta_{i j} & -\rho_{i} q_{i j}(i s) \\
& =\delta_{i j}-a_{i} b_{j}-c_{i} d_{j}-\sum_{m}^{M} e_{i}^{(m)} f_{j}^{(m)} \equiv \sum_{\alpha=1}^{M+2} a_{i}^{(\alpha)} b_{j}^{(\alpha)}
\end{aligned}
$$

with

$$
\begin{array}{rlr}
a_{i} & =\rho_{i} \sigma_{i}^{3} \psi_{1}\left(s \sigma_{i}\right) e^{s \sigma_{i} / 2} \quad b_{j}=A_{j} e^{-s \sigma_{j} / 2}=A_{j}^{0} e^{-s \sigma_{j} / 2}+\frac{\pi}{\Delta} \sum_{n} a_{j}^{(n)} P^{(n)} e^{-s \sigma_{j} / 2} \\
c_{i} & =\rho_{i} \sigma_{i}^{2} \phi_{1}\left(s \sigma_{i}\right) e^{s \sigma_{i} / 2} \quad d_{j}=\beta_{j} e^{-s \sigma_{j} / 2}=\beta_{j}^{0} e^{-s \sigma_{j} / 2}+\frac{2 \pi}{\Delta} \sum_{n} a_{j}^{(n)} \Delta^{(n)} e^{-s \sigma_{j} / 2} \\
e_{i}^{(m)} & =\rho_{i} e^{s \sigma_{i} / 2} \sigma_{i}^{2} \varphi_{1}^{(m)}\left(s \sigma_{i}\right) \quad f_{j}^{(m)}=a_{j}^{(m)} e^{z_{m} \sigma_{j} / 2} \\
\varphi_{1}^{(m)}\left(s \sigma_{i}\right) & =\frac{e^{z_{m} \sigma_{i} / 2}}{s+z_{m}}\left\{\frac{\mathcal{B}_{i}^{(m)}}{z_{m}}-e^{-z_{m} \sigma_{i}}\left(\frac{\mathcal{B}_{i}^{(m)}}{z_{m}}-\delta_{i}^{(m)}\right)\left[1+\frac{z_{m}}{s}\left(1-e^{-s \sigma_{i}}\right)\right]\right\}
\end{array}
$$

Notice that eq.(22) can be written as

$$
\tilde{q}_{i k}^{0^{\prime}}(i s)=-\widetilde{a}_{i} b_{k}-\widetilde{c}_{i} d_{k}-\sum_{m}^{M} \widetilde{e}_{i}^{(m)} f_{k}^{(m)} \equiv \widetilde{\mu}_{i j}=\sum_{\alpha=1}^{M+2} \tilde{a}_{i}^{(\alpha)} b_{j}^{(\alpha)}
$$

with

$$
\begin{aligned}
\tilde{\mathbf{a}}_{i} & =\frac{\left(1+s \sigma_{i} / 2\right)}{s^{2}} e^{-\frac{s \sigma_{i}}{2}} \\
\tilde{\mathbf{c}}_{i} & =\frac{e^{-\frac{s \sigma_{i}}{2}}}{s} \\
\tilde{\mathbf{e}}_{i}^{(m)} & =e^{-\frac{s \sigma_{i}}{2}} \frac{z_{m}}{s+z_{m}} \mathcal{X}_{i}^{(m)} ; \quad \mathcal{X}_{i}^{(m)} \equiv e^{-z_{m} \sigma_{i}}\left(\frac{\mathcal{B}_{i}^{(m)}}{z_{m}}-\delta_{i}^{(m)}\right)
\end{aligned}
$$

With this notation we can rewrite eq.(21) as

$$
2 \pi \tilde{g}_{i j}(s)=\sum_{k} \tilde{\mu}_{i k}\left[\mathcal{M}_{i j}(s)\right]^{-1}
$$

After some algebra we get the general and simple result

$$
2 \pi \tilde{g}_{i j}(s)=-\frac{\widetilde{\mu}_{i j}(s)}{D_{\tau}(s)}
$$

with

$$
D_{\tau}=\operatorname{Det}\left|\delta_{\alpha, \beta}-\left(\sum_{i} a_{i}^{(\alpha)} b_{i}^{(\beta)}\right)\right|
$$


Structure of multi-component/multi-Yukawa mixtures

\section{Selected applications}

We now apply the general result eq.(33) to a few selected examples of interest:

\subsection{The Polydisperse hard spheres mixture}

For hard spheres

$$
\begin{aligned}
& A_{j}=\frac{2 \pi}{\Delta}\left[1+\frac{\pi \zeta_{2}}{2 \Delta} \sigma_{j}\right]=\frac{2 \pi}{\Delta}\left[1+\frac{\zeta_{2}}{2} \beta_{j}\right] \\
& \beta_{j}=\frac{\pi}{\Delta} \sigma_{j}
\end{aligned}
$$

Then we get, using the definitions of eq. (28, 31)

$$
\begin{array}{ll}
a_{i}=\rho_{i} \sigma_{i}^{3} \psi_{1}\left(s \sigma_{i}\right) & b_{j}=A_{j}^{0} \\
c_{i}=\rho_{i} \sigma_{i}^{2} \phi_{1}\left(s \sigma_{i}\right) & d_{j}=\beta_{j}^{0}
\end{array}
$$

and also

$$
\begin{aligned}
\tilde{\mathbf{a}}_{i} & =\left(1+s \sigma_{i} / 2\right) e^{-s \sigma_{i}} / s^{2} \\
\tilde{\mathbf{c}}_{i} & =e^{-s \sigma_{i}} / s
\end{aligned}
$$

The pair correlation function is

$$
\begin{aligned}
& 2 \pi \tilde{g}_{i j}(s)=-\frac{e^{-s \sigma_{i j}}}{s^{2} D_{\tau}}\left\{1+s\left[\left(Q^{H S}\right)_{i j}^{\prime}\left(\sigma_{i j}\right)\right]\right\} \\
& D_{\tau}=1-\sum_{j} \rho_{j} \sigma_{j}^{2}\left\{\sigma_{j} \psi_{1}\left(s \sigma_{j}\right) A_{j}^{0}-\phi_{1}\left(s \sigma_{j}\right) \beta_{j}^{0}+\frac{4 \pi^{2}}{\Delta^{2}} \sum_{i} \rho_{i} \sigma_{i}^{3} \psi_{1}\left(s \sigma_{i}\right) \phi_{1}\left(s \sigma_{j}\right) \lambda_{j i}\right\}
\end{aligned}
$$

\subsection{The electrolyte limit}

We take the limit 27

$$
z_{m}=0
$$

of the general definitions. Then,

$$
\begin{aligned}
a_{i} & =\rho_{i} \sigma_{i}^{3} \psi_{1}\left(s \sigma_{i}\right) \quad b_{j}=A_{j}^{0}+\frac{\pi}{\Delta} a_{j}^{0} P_{n}=A_{j} \\
c_{i} & =\rho_{i} \sigma_{i}^{2} \phi_{1}\left(s \sigma_{i}\right) \quad d_{j}=\beta_{j}^{0}+a_{j}^{0} \Delta_{N}=\beta_{j} \\
e_{i}^{(0)} & =\rho_{i} e^{s \sigma_{i} / 2} \sigma_{i}^{2} \varphi_{1}^{(0)}\left(s \sigma_{i}\right) \quad f_{j}^{(0)}=a_{j}^{(0)} e^{-s \sigma_{j} / 2} \\
\varphi_{1}^{(0)}\left(s \sigma_{i}\right) & =\frac{1}{s}\left\{\frac{\pi}{\Delta} P_{n} \phi_{1}\left(s \sigma_{i}\right)-X_{i}^{(0)}\left[1+\phi_{0}\left(s \sigma_{i}\right)\right]\right\}
\end{aligned}
$$


Structure of multi-component/multi-Yukawa mixtures

Moreover

$$
\begin{aligned}
\tilde{q}_{i j}^{0^{\prime}}(i s) & =\left[\left(1+s \sigma_{i} / 2\right) A_{j}+s \beta_{j}\right] e^{-s \sigma_{i j}} / s^{2}-\sum_{m} \frac{z_{m}}{s+z_{m}} e^{-\left(s+z_{m}\right) \sigma_{i j}} C_{i j}^{(m)} \\
& =\frac{e^{-s \sigma_{i j}}}{s^{2}}\left\{\left[\left(1+s \sigma_{i} / 2\right) A_{j}+s \beta_{j}\right]+s \mathcal{B}_{i}^{(0)} a_{j}^{(0)}\right\} \\
& =\frac{e^{-s \sigma_{i j}}}{s^{2}}\left\{\left[\left(1+s \sigma_{i} / 2\right) A_{j}^{0}+s \beta_{j}^{0}\right]+s \mathcal{X}_{i}^{(0)} a_{j}^{(0)}\right\}
\end{aligned}
$$

and also

$$
\begin{aligned}
\tilde{\mathbf{a}}_{i} & =\frac{\left(1+s \sigma_{i} / 2\right)}{s^{2}} e^{-\frac{s \sigma_{i}}{2}} \\
\tilde{\mathbf{c}}_{i} & =\frac{e^{-\frac{s \sigma_{i}}{2}}}{s} \\
\tilde{\mathbf{e}}_{i}^{(0)} & =\frac{z_{m}}{s+z_{m}} e^{-(s) \sigma_{i} / 2} e^{-z_{m} \sigma_{i}}\left(\hat{\mathcal{B}}_{i}^{(m)}-\delta_{i}^{(m)}\right) \Rightarrow \frac{1}{s} e^{-s \sigma_{i} / 2} \mathcal{B}_{i}^{(0)}
\end{aligned}
$$

so that from eq.(33) we get the symmetric expression (compare [20])

$$
\begin{aligned}
2 \pi \tilde{g}_{i j}(s) & =-\frac{\widetilde{\mu}_{i j}}{D_{\tau}} \\
& =-\frac{e^{-s \sigma_{i j}}}{s^{2} D_{ \pm}}\left\{1+s\left[\frac{\sigma_{i}}{2} A_{j}^{0}+\beta_{j}^{0}+\mathcal{X}_{i}^{(0)} a_{j}^{(0)}\right]\right\}
\end{aligned}
$$

with

$$
D_{ \pm}=\operatorname{Det}\left|\begin{array}{ccc}
1-(a b) & -(a d) & -(a f) \\
-(c b) & 1-(c d) & -(c f) \\
-(e b) & -(e d) & 1-(e f)
\end{array}\right|
$$

where the scalar products ( see also 20]) are defined by

$$
(a b)=\sum_{i} a_{i} b_{i}, \quad(c d)=\sum_{i} c_{i} d_{i}, \quad(e f)=\sum_{i} e_{i} f_{i} \quad \cdots
$$

and we use the definitions of eq.(39).

\section{THE PRIMARY CLOSURE}

The MSA closure condition obtained from Eq.(7) is

$$
2 \pi K \delta_{i}^{(n)} \delta_{j}^{(n)} / z_{n}=\sum_{\ell} D_{i \ell}^{(n)}\left[\delta_{\ell j}-\rho_{\ell} \tilde{q}_{j \ell}\left(i z_{n}\right)\right]
$$

Using the results of the last section we got 34 


$$
\begin{aligned}
& 2 \pi K \delta_{j}^{(n)}+z_{n} \sum_{\ell} \mathcal{I}_{j \ell}^{(n)} a_{\ell}^{(n)} \\
- & \sum_{m} \frac{z_{n}}{z_{n}+z_{m}}\left\{\sum_{k} \rho_{k} a_{k}^{(n)} a_{k}^{(m)}\right\}\left[\sum_{\ell} \mathcal{J}_{j \ell}^{(n)}\left[\Pi_{\ell}^{(m)}-z_{m} X_{\ell}^{(m)}\right]-\mathcal{I}_{j \ell}^{(n)} X_{\ell}^{(m)}\right]=0
\end{aligned}
$$

\section{The 1 Component Case: An explicit continued fraction solution}

In the one component case eq.(46) is simply 34]

$$
-2 \pi K_{n} \rho\left[X_{n}\right]^{2}=z_{n} \beta_{n}\left[1+\sum_{m} \frac{1}{z_{n}+z_{m}} \beta_{m}\right]
$$

with

$$
\begin{aligned}
& X_{n}=\frac{\delta_{n}}{\mathcal{I}^{(n)}+\Gamma_{n} \mathcal{J}^{(n)}} \\
& \beta_{n}=\rho a_{n} X_{n}
\end{aligned}
$$

In the diagonal approximation we get explicit equations for the scaling parameters $\beta_{i}$ [14] ( See appendix III.,[14]) We recall that

$$
\begin{aligned}
& \mathcal{J}^{(n)}=\sigma \phi_{0}\left(z_{n} \sigma\right)+\rho \Delta^{J,(n)} \\
& \Delta^{J,(n)}=-\frac{2 \pi}{\Delta} \sigma^{4} \psi_{1}\left(z_{n} \sigma\right)
\end{aligned}
$$

and

$$
\begin{aligned}
& \mathcal{I}^{(n)}=1+\rho \Delta^{I,(n)} \\
& \Delta^{I,(n)}=\sigma^{2} \phi_{0}\left(z_{n} \sigma\right) \frac{\pi}{2 \Delta} \sigma-\frac{2 \pi}{\Delta} \sigma^{3} \psi_{1}\left(z_{n} \sigma\right)\left[1+\left(\zeta_{2} \frac{\pi}{2 \Delta}\right) \sigma+\sigma z_{n} / 2\right]
\end{aligned}
$$

and we have used the definition (176).

The solution of the $\beta$ equations is obtained solving the linear equation [14]

$$
\vec{\beta}=[\hat{\mathcal{M}}]^{-1} \cdot 2 \vec{\Gamma}
$$

where

$$
\hat{\mathcal{M}}=\left[\begin{array}{cccc}
1 & 1-\gamma_{12} & 1-\gamma_{13} & \cdot \\
1+\gamma_{12} & 1 & 1-\gamma_{23} & \cdot \\
1+\gamma_{13} & 1+\gamma_{23} & 1 & \cdot \\
\cdot & \cdot & \cdot & \cdot
\end{array}\right] ; \quad \vec{\beta}=\left[\begin{array}{c}
\beta_{1} \\
\beta_{2} \\
\cdot
\end{array}\right] ; \quad \vec{\Gamma}=\left[\begin{array}{c}
\Gamma_{1} \\
\Gamma_{2} \\
\cdot
\end{array}\right]
$$


Structure of multi-component/multi-Yukawa mixtures

and

$$
\gamma_{n m}=\frac{2 \Gamma_{n}+z_{n}-2 \Gamma_{m}-z_{m}}{z_{m}+z_{n}}
$$

We give the soultions for the first few cases [14]:

(i) 1 Yukawa $\beta_{1}=2 \Gamma_{1}$

(ii) 2 Yukawas

$$
\beta_{1}=\frac{2 \Gamma_{2}-\beta_{s}}{\gamma_{12}} \quad \beta_{2}=\frac{-2 \Gamma_{1}+\beta_{s}}{\gamma_{12}} \quad \beta_{s}=\frac{2 \Gamma_{2}-2 \Gamma_{1}}{\gamma_{12}}
$$

(iii) 3 Yukawas: This case is slightly more complicated:

The resolvent is

$$
\vec{\mu}^{(3 ; 1)}=\left(\begin{array}{c}
\gamma_{23} \\
\gamma_{31} \\
\gamma_{12}
\end{array}\right)
$$

so that the explicit solution is

$$
\begin{aligned}
& \beta_{1}=\left[\frac{1}{d_{3}}\right]\left[\gamma_{23}\left(\frac{2 \vec{\Gamma} \cdot \vec{\mu}^{(3 ; 1)}}{d_{3}}+s_{23}\right)-z_{23}\right] \\
& \beta_{2}=-\left[\frac{1}{d_{3}}\right]\left[\gamma_{13}\left(\frac{2 \vec{\Gamma} \cdot \vec{\mu}^{(3 ; 1)}}{d_{3}}+s_{13}\right)-z_{13}\right] \\
& \beta_{3}=\left[\frac{1}{d_{3}}\right]\left[\gamma_{12}\left(\frac{2 \vec{\Gamma} \cdot \vec{\mu}^{(3 ; 1)}}{d_{3}}+s_{12}\right)-z_{12}\right]
\end{aligned}
$$

where $d_{3}=\gamma_{12}+\gamma_{23}-\gamma_{13}$.

$$
\beta_{s}=\frac{2 \vec{\Gamma} \cdot \vec{\mu}^{(3 ; 1)}}{d_{3}}
$$

The general case of $n \geq 3$ can be found in the work of Blum et al. [14, 38]

\subsection{The 1 Yukawa case}

In the 1-Yukawa, 1 component case [27] the closure equation is simply

$$
-2 \pi \rho K_{1}\left[X_{1}\right]^{2}=z_{1} \beta_{1}\left[1+\frac{\beta_{1}}{2 z_{1}}\right]
$$

so that putting it all together we get the equation

$$
y_{1}=2 \Gamma_{1}\left(\Gamma_{1}+z_{1}\right)\left[\mathcal{I}^{(1)}+\Gamma_{1} \mathcal{J}^{(1)}\right]^{2} ; \quad y_{1}=-\frac{2 \pi \rho K_{1} \delta_{1}^{2}}{z_{1}}
$$

The physical branch yields the recursion relation

$$
\Gamma_{1}^{(1)}=\frac{y_{1}}{2 z_{1}\left[\mathcal{I}^{(1)}\right]^{2}} ; \quad \Gamma_{1}^{(n+1)}=\frac{y_{1}}{2\left(\Gamma_{1}^{(n)}+z_{1}\right)\left[\mathcal{I}^{(1)}+\Gamma_{1}^{(n)} \mathcal{J}^{(1)}\right]^{2}}
$$


Structure of multi-component/multi-Yukawa mixtures

For large $z_{1} \geq 3$, we may disregard the exponential terms in Eqs(176), and then we get the simple

$$
\mathcal{I}^{(1)}=1+\frac{2 \pi \rho\left(1+\frac{\pi \rho}{3}-\frac{\pi z_{1} \rho}{4}\right)}{z_{1}^{3} \Delta^{2}} ; \quad \mathcal{J}^{(1)}=\frac{1}{z_{1}}+\frac{\pi \rho\left(-2+z_{1}\right)}{z_{1}^{3} \Delta}
$$

from where

$$
\Gamma_{1}=\frac{y_{1}}{2 z_{1}\left(1-\frac{\pi^{2} \rho^{2}}{2 z_{1}^{2} \Delta^{2}}+\frac{2 \pi \rho(3+\pi \rho)}{3 z_{1}^{3} \Delta^{2}}\right)^{2}} ; \quad \Gamma_{2}=\frac{y_{1}}{2\left(\Gamma_{1}+z_{1}\right) \mathcal{D}_{\Gamma, 1}^{2}}
$$

with

$$
\mathcal{D}_{\Gamma, 1}=1+\frac{1}{z_{1}^{2} \Delta}\left[\frac{-\left(\pi^{2} \rho^{2}\right)}{2 \Delta}+\frac{2 \pi \rho\left(1+\frac{\pi \rho}{3}\right)}{z_{1} \Delta}-\frac{2 y_{1}\left(-\Delta+\frac{2 \pi \rho\left(2-z_{1}\right)}{z_{1}^{2}}\right)}{\left(2-\frac{\pi^{2} \rho^{2}}{z_{1}^{2} \Delta^{2}}+\frac{4 \pi \rho\left(1+\frac{\pi \rho}{3}\right)}{z_{1}^{3} \Delta^{2}}\right)^{2}}\right]
$$

The solution is always convergent for the atractive case, and we do get the Onsagerian limits for large densities and zero temperature correctly. It has been successfully tested numerically against other numerical methods [37] and Hernando, (unpublished, but using Pastore's criterion). For the repulsive case $K>0$ this equation has poles and unphysical divergencies occurring when the Onsagerian limits are attained. These divergencies are removed when a suitable reference hard core is introduced.

\subsection{The multiyukawa case}

The above solution is valid for any number of Yukawas $n \geq 1$. We write Eq. (47) in matrix form:

$$
\left[\begin{array}{c}
y_{1} \\
y_{2} \\
\cdot
\end{array}\right]=\left[\begin{array}{ccc}
1+\beta_{1} / s_{11} & \beta_{1} / s_{12} & \cdot \\
\beta_{2} / s_{12} & 1+\beta_{2} / s_{22} & \cdot \\
\cdot & \cdot & \cdot
\end{array}\right]\left[\begin{array}{c}
\beta_{1} \\
\beta_{2} \\
\cdot
\end{array}\right]
$$

Using equations (54) and (55) we find

$$
\vec{\Gamma}=\frac{1}{2} \hat{\mathcal{M}} \cdot\left[\begin{array}{ccc}
1+\beta_{1} / s_{11} & \beta_{1} / s_{12} & \cdot \\
\beta_{2} / s_{12} & 1+\beta_{2} / s_{22} & \cdot \\
\cdot & \cdot & \cdot
\end{array}\right]^{-1} \cdot\left[\begin{array}{c}
y_{1} /\left[\mathcal{I}^{(1)}+\Gamma_{1} \mathcal{J}^{(1)}\right]^{2} \\
y_{2} /\left[\mathcal{I}^{(2)}+\Gamma_{2} \mathcal{J}^{(2)}\right]^{2} \\
\cdot
\end{array}\right]
$$

with

$$
s_{n m}=z_{n}+z_{m}
$$

This relation is the extension of Eq. (63) to the multiyukawa case: The iteration follows exactly the same steps, only that now we have to use the extra equation (154): The first iterate is

$$
\vec{\Gamma}^{(0)}=\frac{1}{2}\left[\begin{array}{cccc}
1 & 1-\frac{z_{12}}{s_{12}} & 1-\frac{z_{13}}{s_{13}} & \cdot \\
1+\frac{z_{12}}{s_{12}} & 1 & 1-\frac{z_{23}}{s_{23}} & \cdot \\
1+\frac{z_{13}}{s_{13}} & 1+\frac{z_{23}}{s_{23}} & 1 & \cdot \\
\cdot & \cdot & \cdot & \cdot
\end{array}\right] \cdot\left[\begin{array}{c}
y_{1} /\left[\mathcal{I}^{(1)}\right]^{2} \\
y_{2} /\left[\mathcal{I}^{(2)}\right]^{2} \\
\cdot
\end{array}\right] ; \quad z_{n m}=z_{n}-z_{m}
$$


and then

$$
\vec{\Gamma}^{(n+1)}=\frac{1}{2} \hat{\mathcal{M}}^{(n)} \cdot\left[\left\{\begin{array}{ccc}
1+\beta_{1} / s_{11} & \beta_{1} / s_{12} & \cdot \\
\beta_{2} / s_{12} & 1+\beta_{2} / s_{22} & \cdot \\
\cdot & \cdot & \cdot
\end{array}\right\}^{(n)}\right]^{-1} \cdot\left[\begin{array}{c}
y_{1} /\left[\mathcal{I}^{(1)}+\Gamma_{1}^{(n)} \mathcal{J}^{(1)}\right]^{2} \\
y_{2} /\left[\mathcal{I}^{(2)}+\Gamma_{2}^{(n)} \mathcal{J}^{(2)}\right]^{2} \\
\cdot
\end{array}\right]
$$

where the superscript $(n)$ indicates the level of the iteration.

\section{Summary of results}

- A new formulation of the pair distribution function for a large class of systems represented by a multiyukawa closure of the Ornstein-Zernike equation (11) which includes systems such as water, ionic mixtures, ion channels and polymers is presented. This formulation is considerably simpler and more explicit than those in the literature because it involves directly measurable parameters such as the contact pair distribution function. It is also capable of handling the new improved versions of the MSA and the realistic octupolar model of water [7].

- The new result shown in equation (2) is simple and explicit: For example in the case of the SANS and SAXS experiments [1, 2] our result show explicitly the connection between the number of peaks in the diffraction spectra to the theoretical formula. because we get for dilute systems a sum of M Lorentzians, one for each yukawa term in the closure.

- The one component case has been discussed in several papers, largely in collaboration with J. Hernando [6], although most of the numerical results are still unpublished. The conclusion is that once the branch points are identified the continued fraction formalism always converges ( see also 37]).

\section{Appendix I: The algebraic solution of the general Yukawa closure of the Ornstein Zernike equation}

We quote the results from the review of Blum and Hernando [34]: The solution of the system of equations (1920) yields

$$
A_{j}=A_{j}^{0}+\frac{\pi}{\Delta} \sum_{n} a_{j}^{(n)} P^{(n)} ; \quad \beta_{j}=\beta_{j}^{0}+\frac{2 \pi}{\Delta} \sum_{n} a_{j}^{(n)} \Delta^{(n)}
$$

where

$$
\begin{aligned}
& A_{j}^{0}=\frac{2 \pi}{\Delta}\left[1+(1 / 2) \zeta_{2} \frac{\pi}{\Delta} \sigma_{j}\right] ; \quad \beta_{j}^{0}=\frac{\pi}{\Delta} \sigma_{j} \\
& \frac{\pi}{\Delta} P^{(n)}=\frac{1}{z_{n}} \sum_{\ell} \rho_{\ell}\left[A_{\ell}^{0} X_{\ell}^{(n)}+2 \beta_{\ell}^{0}\left(z_{n} X_{\ell}^{(n)}-\Pi_{\ell}^{(n)}\right)\right] \\
& \Delta^{(n)}=-\frac{1}{z_{n}^{2}} \sum_{\ell} \rho_{\ell}\left[X_{\ell}^{(n)}\left(A_{\ell}^{0}-z_{n} \beta_{\ell}^{0}\right)+2 \beta_{\ell}^{0}\left(z_{n} X_{\ell}^{(n)}-\Pi_{\ell}^{(n)}\right)\right]
\end{aligned}
$$


Structure of multi-component/multi-Yukawa mixtures

We have

$$
\begin{aligned}
& \Pi_{j}^{(n)}=\hat{B}_{j}^{(n)}+\Delta^{(n)}+\frac{\pi}{2 \Delta} \sigma_{j} P^{(n)} \\
& X_{i}^{(n)}=\delta_{i}^{(n)}+\sigma_{i} \hat{B}_{i}^{(n)} \phi_{0}\left(z_{n} \sigma_{i}\right)+\sigma_{i} \Delta^{(n)} \\
& \Pi_{j}^{(n)}=\hat{\xi}_{j}^{(n)}+\sum_{\ell} \hat{\mathcal{I}}_{j \ell}^{(n)} \hat{B}_{\ell}^{(n)} \\
& X_{j}^{(n)}=\gamma_{j}^{(n)}+\sum_{\ell} \hat{\mathcal{J}}_{j \ell}^{(n)} \hat{B}_{\ell}^{(n)}
\end{aligned}
$$

where

$$
\begin{aligned}
\hat{\mathcal{I}}_{j \ell}^{(n)} & =\delta_{j \ell}-\rho_{\ell} \sigma_{\ell}^{2}\left[\beta_{j}^{0} \phi_{1}\left(z_{n} \sigma_{\ell}\right)+A_{j}^{0} \sigma_{\ell} \psi_{1}\left(z_{n} \sigma_{\ell}\right)\right] \\
\hat{\mathcal{J}}_{j \ell}^{(n)} & =\delta_{j \ell} \sigma_{j} \phi_{0}\left(z_{n} \sigma_{\ell}\right)-2 \rho_{\ell} \beta_{j}^{0} \sigma_{\ell}^{3} \psi_{1}\left(z_{n} \sigma_{\ell}\right)
\end{aligned}
$$

with

$$
\begin{aligned}
& \hat{\xi}_{j}^{(n)}=-\frac{1}{z_{n}^{2}} \sum_{\ell} \rho_{\ell} \delta_{\ell}^{(n)}\left[A_{j}^{0}+z_{n} Q^{\prime}\left(\sigma_{\ell j}\right)\right] \\
& \hat{\gamma}_{j}^{(n)}=\delta_{j}^{(n)}-\frac{2 \pi \sigma_{j}}{z_{n}^{2} \Delta} \sum_{\ell} \rho_{\ell} \delta_{\ell}^{(n)}\left(1+\frac{z_{n} \sigma_{\ell}}{2}\right)
\end{aligned}
$$

Here we define [34]:

$$
\begin{aligned}
& \zeta_{n}=\sum_{k} \rho_{k} \sigma_{k}^{n} \\
& \Delta=1-\pi \zeta_{3} / 6 \\
& \tilde{g}_{i j}(s)=\int_{0}^{\infty} d r r g_{i j}(r) e^{-s r} \\
& \gamma_{i j}^{(n)}=2 \pi \tilde{g}_{i j}\left(z_{n}\right) \rho_{j} / z_{n} \\
& \hat{B}_{j}^{(n)}=\sum_{i} z_{n} \delta_{i}^{(n)} \gamma_{j i}^{(n)} e^{z_{n} \sigma_{i j}} \\
& \psi_{1}\left(s \sigma_{i}\right)=\frac{1}{2}\left[\gamma_{z}\left(3, s \sigma_{i}\right)-\gamma_{z}\left(2, s \sigma_{i}\right)\right] \\
& \phi_{1}\left(s \sigma_{i}\right)=\left[\gamma_{z}\left(2, s \sigma_{i}\right)-\gamma_{z}\left(1, s \sigma_{i}\right)\right]
\end{aligned}
$$

with the incomplete $\gamma$ function

$$
\gamma_{z}(n, z)=\frac{(n-1) !}{z^{n}}\left[1-e^{-z} \sum_{i}^{n-1} \frac{z^{i}}{i !}\right]
$$


Structure of multi-component/multi-Yukawa mixtures

9. Appendix II: Inverse of $\mathbf{M}_{i j}(s)$

$$
\widehat{\mathbf{M}}_{i j}(s)=\delta_{i j}-\mu_{i j} ; \quad \mu_{i j}=\sum_{\alpha=1}^{\alpha_{m}} a_{i}^{(\alpha)} b_{j}^{(\alpha)}
$$

The determinant of this matrix can be expanded in its minors $\mathcal{M}_{i j}$

$$
D_{T}=\operatorname{Det}\left|\delta_{i j}-\mu_{i j}\right|=\sum_{i}\left[\delta_{i j}-\mu_{i j}\right](-)^{i+j} \mathcal{M}_{i j} \quad ; \quad \forall j
$$

This determinant can also be condensed into a simpler and more compact form

$$
D_{T}=D_{\tau}=\left|\delta_{\alpha, \beta}-\left(\sum_{i} a_{i}^{(\alpha)} b_{i}^{(\beta)}\right)\right|
$$

which can also be expanded in its minors $\mathbf{M}^{(\alpha, \beta)}$

$$
\begin{aligned}
D_{\tau} & =\sum_{\beta}\left(\delta_{\alpha, \beta}-a^{(\alpha)} \bullet b^{(\beta)}\right)[-]^{\alpha+\beta} \mathbf{M}^{(\alpha, \beta)} ; \quad \forall \alpha \\
& =\sum_{\beta}\left(\delta_{\alpha, \beta}-a^{(\alpha)} \bullet b^{(\beta)}\right) \mathcal{A}^{(\alpha, \beta)} ; \quad \mathcal{A}^{(\alpha, \beta)}=[-]^{\alpha+\beta} \mathbf{M}^{(\alpha, \beta)}
\end{aligned}
$$

Using Cramer's rule then we find the general inverse

$$
\begin{array}{ll}
{\left[\mathbf{M}^{-1}(s)\right]_{i j}=\delta_{i j}+\frac{1}{D_{\tau}} \sum_{\beta} a_{i}^{(\alpha)} b_{j}^{(\beta)}\left\{\mathcal{A}^{(\alpha, \beta)}\right\} ;} & \forall\{\alpha, \beta\} \\
\sum_{k}\left\{\delta_{i k}-\mu_{i k}\right\} \cdot\left[\mathbf{M}^{-1}(s)\right]_{k j} & =\delta_{i j} \\
\sum_{k}\left\{\delta_{i k}-\mu_{i k}\right\} \cdot\left[\delta_{i j}+\frac{1}{D_{\tau}} \sum_{\beta}(-)^{\alpha+\beta} a_{k}^{(\alpha)} b_{j}^{(\beta)}\left\{\mathcal{M}^{(\alpha, \beta)}\right\}\right] & =\delta_{i j} \\
\sum_{k}\left[\sum_{\gamma} a_{i}^{(\gamma)} b_{k}^{(\gamma)}\right] \cdot\left[\frac{1}{D_{\tau}} \sum_{\beta} a_{k}^{(\alpha)} b_{j}^{(\beta)}\left\{\mathcal{A}^{(\alpha, \beta)}\right\}\right] & =\mu_{i j}
\end{array}
$$

which means that we must satisfy

$$
\begin{aligned}
\mu_{i j}= & \sum_{\gamma, \beta} a_{i}^{(\gamma)} b_{j}^{(\gamma)}\left[\frac{1}{D_{\tau}} \sum_{k} b_{k}^{(\gamma)} a_{k}^{(\alpha)}\right] \mathcal{A}^{(\alpha, \beta)} \\
& \sum_{k}\left(\mu_{i k}\left[\mathbf{M}^{-1}(s)\right]_{i j}^{\alpha, \beta}\right)=\sum_{\alpha} a_{i}^{(\alpha)} \sum_{k} b_{k}^{(\beta)}\left(-\frac{1}{D_{\tau}} \sum_{\gamma} a_{k}^{(\gamma)} b_{j}^{(\delta)}\left\{\mathcal{A}_{\gamma, \delta}\right\}\right) ; \quad \forall \delta \\
= & \left.\frac{1}{D_{\tau}} \sum_{\beta}(-)^{\alpha+\beta} a_{i}^{(\alpha)} b_{j}^{(\beta)}\left\{\mathcal{M}^{(\alpha, \beta)}\right\}\right]+\sum_{\alpha} a_{i}^{(\alpha)}\left(-\frac{1}{D_{\tau}} \sum_{\gamma}\left[\sum_{k} b_{k}^{(\beta)} a_{k}^{(\gamma)}\right]\left\{\mathcal{A}_{\gamma, \delta}\right\}\right) b_{j}^{(\delta)} ; \\
= & \mu_{i j}
\end{aligned}
$$

as it should 


\section{Acknowledgements}

Support from NSF through grant DMR02-03755 and DOE grant DE-FG02-03ER 15422 is acknowledged.

We wish Prof. Sow Hsin Chen a very happy and healthy 70th birthday. The subject of this paper was inspired in conversationss with him.

[1] Liu, Y. Chen, W.-R. and Chen, S.H.,2005, J. Chem. Phys.122, 044507.

[2] Broccio, M ., Costa, D. Liu, Y. and Chen, S.H., Preprint (2005).

[3] Rosenfeld, Y. and Blum, L., 1986, J. Chem. Phys. , 851556.

[4] Blum, L. and Narten, A.H., 1972, J. Chem. Phys., 56 , 5197.

[5] Narten, A.H., Blum L., and Fowler, R. H., 1974, J. Chem. Phys., 603378.

[6] Blum, L. and Hernando, J. A., 2001, Condensed Matter Theories,16,411, S. Hernandez and J. W. Clark, editors, Nova Publishers New York.

[7] Blum, L., Vericat F. and Degreve L. , Physica A 265 , 396.

[8] Enriquez, A. and Blum, L., 2005, Mol. Phys. , 1033201.

[9] Blum, L., and Bernard, O., 2004, Proc. International School of Physics "Enrico Fermi", Course $C L V$, Mallamace, F. and Stanley, H. E., Editors, IOS Press, Amsterdam, 155, 335.

[10] Lebowitz, J.L., 1964, Phys. Revs, 1964, 133, A895.

[11] Blum, L. and Stell, G., 1979, J. Chem. Phys.71 42.

[12] Blum, L. and Hoye, J. S.,1977, J. Phys. Chem., 81 ,1311.

[13] Blum, L. and Herrera J.N., 1999 Mol. Phys. 96, 821.

[14] Blum, L. and Ubriaco, M., 2000, Mol. Phys., 98, 829.

[15] Percus J.K. and Yevick G.,1964, Phys. Revs. ,110 250 .

[16] Lebowitz, J.L. , and Percus, J.K., 1966, Phys. Revs,144, 251.

[17] Lin, Y.Z. Li, Y.G. Lu Y. F., and Liu, Z. P. 2002, J. Chem. Phys.117 10165.

[18] Wu, C. F. and Chen, S.H., 1987, J. Chem. Phys., 87, 6199.

[19] Wu, C. F. and Chen, S.H., 1988, Biopolymers, 27, 1065.

[20] Blum L. and Høye J.S., 1978, J. Stat. Phys.,19 , 317.

[21] Morales, V. Anta, J.A. and Lago, S. 2003, Langmuir ,19, 475.

[22] Kaldasch, J., Laven J., and Stein, H. N. 1996, Langmuir ,12, 6197.

[23] Victor, J.M. and Hansen, J.P.,1985,J. Chem. Soc., Faraday Trans.2-81, 43 .

[24] Grimson, M. J., J. Chem. Soc.,1982,J. Faraday Trans. 2 79, 817.

[25] Verwey, E. J. W. and Overbeek, J. TH. G. , 1948, Theory of the Stability of Lyophobic Colloids, Elsevier, Amsterdam.

[26] Blum L., Vericat F. and Herrera, J.N.,1992, J. Stat. Phys.,66, 249.

[27] Blum L. , 1980, J. Stat. Phys.,22 ,661.

[28] Blum L., 1975, Mol. Phys.30, 1529 .

[29] Ginoza M., 1986, J.Phys. Soc. Japan,55, 95 .

[30] Ginoza M., 1986, J.Phys. Soc. Japan ,55, 1782.

[31] Ginoza M., 1990, Mol. Phys.,71, 145.

[32] Ginoza M. and Yasutomi M.,1998, J. Stat. Phys.,90, 1475.

[33] Blum L. and Herrera J.N.,1998 Mol. Phys. , 95, 425.

[34] Blum L. and Hernando, J. A., 2002, J. of Phys.: Cond. Matt. 1410933 .

[35] Pastore, G., 1988, Mol. Phys. , 63, 731.

[36] Hernando, J. A., and Blum L., 1993, J. of Phys.: Cond. Matt., 5 Suppl. 33A, A109.

[37] Vazquez, O. R., Herrera, J.N. and Blum, L., 2003, Physica A, 325319 .

[38] Blum, L. and Ubriaco, M.,2000, Physica A, 279224. 